\title{
A 1310/1550 nm Fully-Integrated Optical Receiver with Schottky Photodiode and Low-Noise Transimpedance Amplifier in $40 \mathrm{~nm}$ Bulk CMOS
}

\author{
Wouter Diels, Michiel Steyaert and Filip Tavernier \\ Department of Electrical Engineering, Katholieke Universiteit Leuven \\ 3001 Leuven, Belgium
}

\begin{abstract}
This paper presents an optical receiver in $40 \mathrm{~nm}$ bulk CMOS for 1310 and $1550 \mathrm{~nm}$ light, which is used for singlemode fiber communication. A Schottky photodiode converts the light into a current with a responsivity of 1 and $0.3 \mathrm{~mA} / \mathrm{W}$ respectively. A 3-stage voltage amplifier with negative resistive and positive capacitive feedback is used as transimpedance amplifier with a simulated integrated input-referred noise current of less than 100 and $200 \mathrm{nA}$ for a bandwidth of 0.5 and $1 \mathrm{GHz}$ respectively. Measurement results are presented of the chip, which consumes $48.7 \mathrm{~mW}$, for both 1310 and $1550 \mathrm{~nm}$ light. For $1310 \mathrm{~nm}$ light, bit rates up to $1 \mathrm{~Gb} / \mathrm{s}$ are received with a bit error rate of $10^{-10}$ and a sensitivity of $3.4 \mathrm{dBm}$. To the authors' best knowledge, this is the first fully-integrated optical receiver in bulk CMOS for these wavelengths.
\end{abstract}

\section{INTRODUCTION}

Although occasionally outperformed by other technologies in some aspects, CMOS remains a popular choice for commercial electronic applications, due to its excellent costeffectiveness and massive integration possibilities. However, high-speed optical communications remains a domain in which dedicated technologies are being preferred, although research in fully-integrated CMOS solutions has taken strides forward. Most research in this domain has been situated in the receiver side, with the photodiode being integrated with the analog front-end circuitry, such as transimpedance amplifiers (TIAs) and equalizers. Traditionally, the fully-integrated receivers are conceived for $850 \mathrm{~nm}$ multi-mode fiber communication, as the photoelectric effect still occurs for this wavelength in silicon [1], [2]. While the integrated P-substrate/N-well photodiodes have a quite high quantum efficiency for light modulated at low frequencies, the performance already starts decaying at frequencies in the MHz range. This is due to the low absorption coefficient of silicon at this wavelength, resulting in the majority of generated electron-hole pairs being generated far from the diode junction. They have to slowly diffuse upwards in order to be collected. Still, for modulation frequencies in the lower $\mathrm{GHz}$ range, responsivities of $20 \mathrm{~mA} / \mathrm{W}$ are not uncommon [2]. The frequency roll-off of the photocurrent response does lead to intersymbol interference (ISI), for which 2 type of solutions have been demonstrated. Using a spatially modulated light photodiode, bandwidth can be exchanged for responsivity [3]. Alternatively, the limited optical photocurrent bandwidth can be compensated electrically by implementing equalizers
[4], again demonstrating the power of CMOS integration. Recently, it has been shown that the $1310 / 1550 \mathrm{~nm}$ wavelengths used in single-mode communication can also be detected in CMOS processes by means of Schottky photodiodes [5], [6]. When light is absorbed in the metal-like silicide layers and the resulting excited electrons have sufficient energy to cross the Schottky energy barrier, these devices can detect photons with energies below the silicon band gap. Theory predicts that the resulting photocurrent has a very high optical bandwidth (order of $10^{10} \mathrm{~Hz}$ ). However, the reported responsivities are in the order of $1 \mathrm{~mA} / \mathrm{W}$. As such, in order for these photodiodes to be used in a fully-integrated optical receiver, the TIA should minimize the added noise. In [7], we presented the first $1310 \mathrm{~nm}$ Schottky optical receiver in bulk CMOS fabricated in a standard $40 \mathrm{~nm}$ process. The chip can receive $1310 \mathrm{~nm}$ data rates up to $1 \mathrm{~Gb} / \mathrm{s}$ for a bit error rate of $10^{-10}$. However, no details about the circuit design were given. In this paper, we discuss the design much more extensively and also present additional measurement results, including $1550 \mathrm{~nm}$ light performance.

This paper is structured as follows. Section II discusses the receiver blocks, such as the Schottky photodiode and the TIA. Section III presents the measurement results, while Section IV gives a conclusion.

\section{RECEIVER ARCHITECTURE}

The fully-differential circuit can be seen in Fig. 1. Two identical Schottky photodiodes are AC-coupled to the TIA. Only one photodiode is illuminated and the input TIA current thus contains a significant common mode component. The TIA should therefore only amplify differential signals and attenuate common mode signals. Furthermore, as a nonreturn-to-zero (NRZ) optical signal inherently also contains a DC component, AC coupling was preferred to prevent this DC current reaching the TIA and potentially causing biasing problems in the subsequent stages. The output of the TIA is connected to a limiting amplifier (LA), whose output is connected to an output buffer. It should be noted that an equalizer was deliberately not included in the design to demonstrate the photodiode's high optical bandwidth. In the following subsections, the photodiode and the analog front-end design are discussed. 


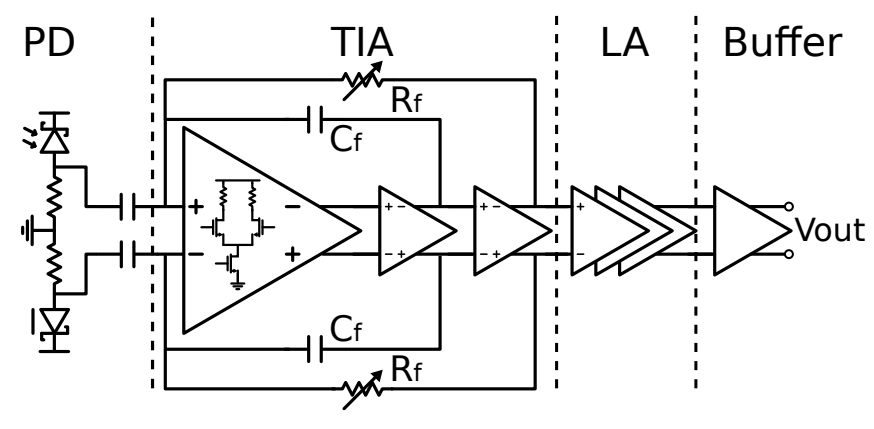

Fig. 1: Block diagram of the presented $1310 / 1550 \mathrm{~nm}$ fullyintegrated optical receiver

\section{A. Schottky photodiode}

A cross-section of the receiver Schottky photodiode can be seen in Fig. 2. In bulk CMOS processes, Schottky diodes can be realized by placing contacts to lowly doped silicon, such as in N-well, P-well or P-substrate regions. The Schottky junction itself is formed between silicide and silicon. In this work, $\mathrm{N}$-well Schottky diodes were preferred, as these have a significantly smaller dark current than their P-well counterpart in this technology [5]. Schottky diodes can detect photons with energy below the band gap energy of silicon through a mechanism called internal photoemission (IPE). When photons with at least the Schottky barrier energy are absorbed in a metal or metal-like silicide layer, the resulting excited electrons can cross the Schottky junction and contribute to the photocurrent. As this process nearly occurs in a plane, the theoretical optical bandwidth of IPE is very high. However, as the majority of light is reflected, the quantum efficiency for surface-illuminated devices tends to be lower than $1 \%$ [5]. For CMOS Schottky photodiodes, the best responsivities are obtained when the chip is illuminated from the substrate side (backside illumination), as photons are absorbed closest to the junction. The IPE quantum efficiency increases for shorter wavelengths, as demonstrated in [6]. For this design, a distributed layout was preferred, in which photosensitive Schottky regions are periodically surrounded by Ohmic strips as illustrated in Fig. 2. Ohmic and Schottky regions are isolated by shallow trench isolation (STI).

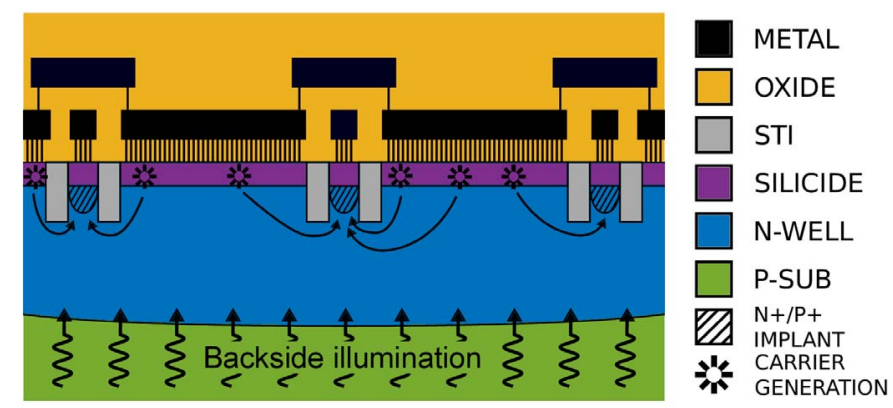

Fig. 2: Cross-section of the photodiode layout
While the reduced fill factor of this type of layout results in a lower responsivity, it also decreases the capacitance and significantly reduces the series resistance, which maximizes the device's electrical bandwidth and minimizes its thermal noise.

The photodiode has a diameter of $26 \mu \mathrm{m}$ and a Schottky region area of $270 \mu^{2}$. During the design phase, the photodiode was electrically modeled by mapping the measurement results of [5] to this photodiode shape. The model's validity was confirmed afterwards as the photodiode was also fabricated separately. The measured dark current, capacitance and $1310 / 1550 \mathrm{~nm}$ responsivities in function of reverse voltage can be seen in Fig. 3 .

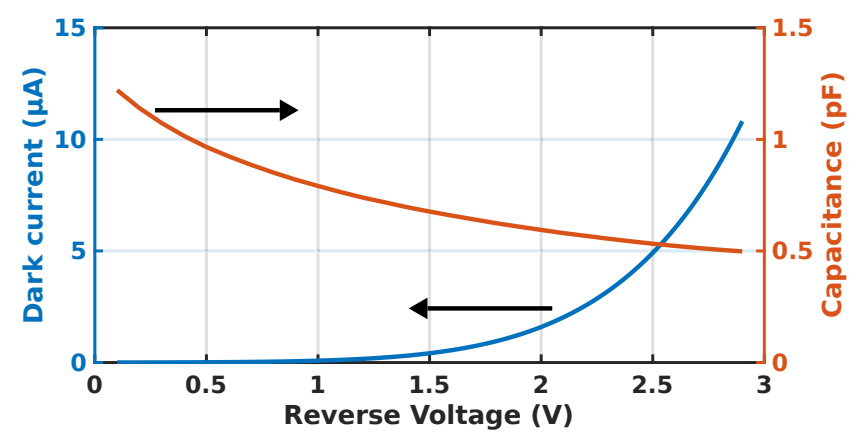

(a)

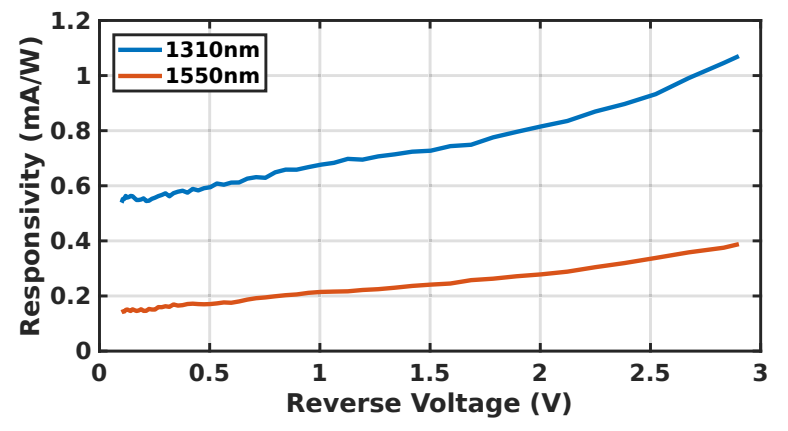

(b)

Fig. 3: Measured photodiode characteristics vs. reverse voltage (a) Dark current and capacitance (b) 1310/1550 nm responsivities

\section{B. Circuit design and considerations}

As the responsivity is around $1 \mathrm{~mA} / \mathrm{W}$ for $1310 \mathrm{~nm}$ light (Fig. 3b), input currents of no more than a few micro amperes are to be expected for average optical input powers near $0 \mathrm{dBm}$. Therefore, when NRZ optical data is applied, the TIA must have an input-referred noise current of less than $100 \mathrm{nA}$ for the receiver to have a bit error rate (BER) below $10^{-9}$. The TIA is a 3-stage amplifier in resistive negative feedback $\left(R_{f}\right)$ and capacitive positive feedback $\left(C_{f}\right)$ configuration. Each amplifier stage is a wideband resistively loaded differential pair with current source. Expressions for the TIA bandwidth BW, equivalent input capacitance $C_{i n, e q}$ and transimpedance gain $Z_{t i a}$ can be found in (1) to (3), where $A_{0, x-y}$ 
is the cascaded open-loop voltage gain of stages $\mathrm{x}$ to $\mathrm{y}, R_{f}$ is the feedback resistance, $C_{p d}$ is the photodiode capacitance, $C_{i n, x}$ is the input capacitance of stage x, $C_{f}$ is the feedback capacitance and $R_{o u t, x}$ is the output resistance of stage $\mathrm{x}$.

$$
\begin{gathered}
B W=\frac{A_{0,1-3}}{2 \pi R_{f} C_{i n, e q}} \\
C_{i n, e q}=C_{p d}+C_{i n, 1}-C_{f}\left(1+A_{0,1-2}\right) \\
Z_{t i a}=\frac{A_{0,1-3} R_{f}-R_{o u t, 3}}{1+A_{0,1-3}}
\end{gathered}
$$

The dominant pole of the TIA is located at its input. The capacitive feedback between the input and the 2nd stage output results in a negative capacitance on the input node of the TIA, thus decreasing the overall input capacitance on this node, which is dominated by the photodiode and 1st amplifier stage input capacitances. This means that the feedback resistance can be higher for the same bandwidth, which increases the transimpedance gain, which is roughly $R_{f}$. However, conversely, the positive capacitive feedback results in additional positive capacitance at the 2nd stage output node, resulting in decreased loop-gain phase margin. The output noise can be attributed to two white noise sources in the TIA: the feedback resistance and the equivalent inputreferred noise voltage of the open-loop amplifier. Increasing the feedback resistance for a constant bandwidth results in an increase of the feedback resistance noise contribution at the output. While this portion of the integrated output noise power increases linearly with $R_{f}$, it must be noted that the output signal power is proportional with $Z_{t i a}^{2}$. The TIA thus gains SNR at the cost of stability with the positive capacitive feedback technique. The noise contribution of the amplifier can be minimized by having a large transconductance in the first stage. Therefore, the first amplifier stage is 4 times larger than the other 2 stages. The sizing of the stages is summarized in TABLE I. A $150 \mathrm{fF}$ MOM-capacitor implements the capacitive feedback, whereas an NMOS in the triode region is used as feedback resistor. The feedback resistance can thus be tuned by changing its gate voltage, providing the flexibility to exchange bandwidth for transimpedance gain. Fig. 4 shows the simulated integrated input-referred noise current and transimpedance gain in function of the TIA bandwidth. These curves were obtained by sweeping the feedback resistor gate voltage. As can be seen, integrated input-referred noise currents below 100 and $200 \mathrm{nA}$ can be obtained for bandwidths lower than 0.5 and $1 \mathrm{GHz}$ respectively, enabling $\mathrm{Gb} / \mathrm{s}$ performance for $1310 \mathrm{~nm}$ light.

The TIA converts the input current into a voltage with an amplitude in the order of a few millivolts. These signals are not sufficiently large for a signal processing block such as a clock and data recovery. The voltage is therefore amplified further in a limiting amplifier (LA). It consists of 3 stages identical to the last stages of voltage amplifier in the TIA. Finally, the signals are buffered with a CML buffer to be measured with $50 \Omega$ terminated instruments.

\begin{tabular}{c|c|c|c}
\hline Stage & 1 & 2 & 3 \\
\hline Differential pair size & $\frac{684 \mu \mathrm{m}}{40 \mathrm{~nm}}$ & $\frac{171 \mu \mathrm{m}}{40 \mathrm{~nm}}$ & $\frac{171 \mu \mathrm{m}}{40 \mathrm{~nm}}$ \\
& $\frac{900 \mu \mathrm{m}}{100 \mathrm{~nm}}$ & $\frac{225 \mu \mathrm{m}}{100 \mathrm{~nm}}$ & $\frac{225 \mu \mathrm{m}}{100 \mathrm{~nm}}$ \\
Current source size & $40 \Omega$ & $160 \Omega$ & $160 \Omega$ \\
Load resistance & $380 \mathrm{fF}$ & $95 \mathrm{fF}$ & $95 \mathrm{fF}$ \\
Input capacitance & $130 \mathrm{mS}$ & $32.5 \mathrm{mS}$ & $32.5 \mathrm{mS}$ \\
Differential pair gm & $15.2 \mathrm{~mA}$ & $3.8 \mathrm{~mA}$ & $3.8 \mathrm{~mA}$ \\
Current & 3 & 3 & 3 \\
Gain & $10 \mathrm{GHz}$ & $9.1 \mathrm{GHz}$ & $9.1 \mathrm{GHz}$ \\
Bandwidth &
\end{tabular}

TABLE I: Open-loop amplifier stages simulation summary

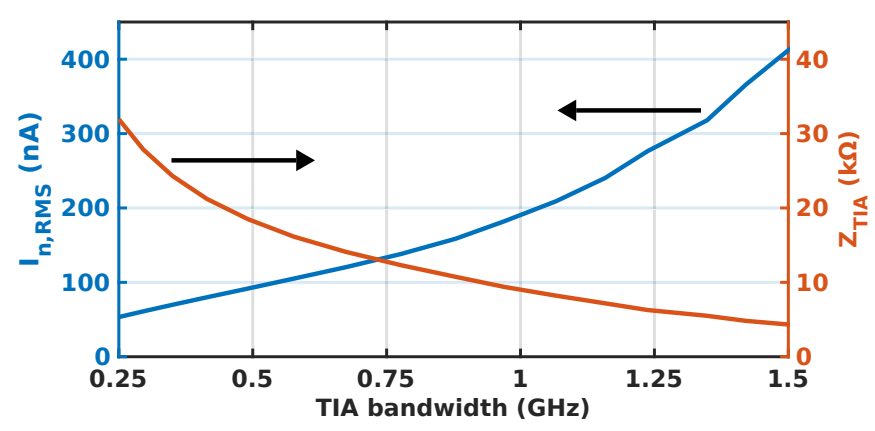

Fig. 4: Simulated TIA input referred current noise and transimpedance gain vs. TIA bandwidth

\section{Measurements}

The fully integrated optical receiver was fabricated in a standard $40 \mathrm{~nm}$ bulk CMOS process. The chip photograph can be seen in Fig. 5. The chip has a size of $510 \mu \mathrm{m}^{2} \times 665 \mu^{2}$, while the core area is only $120 \mu^{2} \times 210 \mu \mathrm{m}^{2}$. For measurements, the die was flip chip bonded on a PCB to facilitate backside illumination. On a supply voltage of $0.95 \mathrm{~V}$, the TIA and LA combined consume $48.7 \mathrm{~mW}$. For measurements, the photodiode was reverse biased at $2.5 \mathrm{~V}$ as this yields favorable capacitance and responsivity for sufficiently low dark currents.

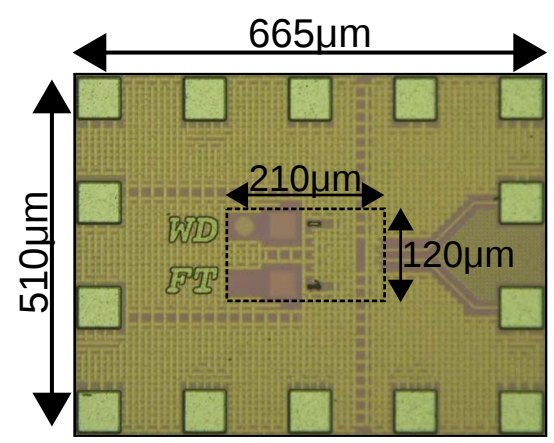

Fig. 5: Chip photograph of the optical receiver

A small-signal measurement of S21-parameters is displayed in Fig. 6 for different values of the tunable feedback resistor. For this measurement, port 1 of the VNA is connected to the laser driver, while port 2 is connected to the output of the receiver. The modulation current of the laser driver is set sufficiently low to observe small-signal behavior in the 
full receiver chain. The S21-parameters have been normalized to the highest value at low frequencies. It can be observed that by tuning the feedback resistor, transimpedance gain and bandwidth can be exchanged over almost 2 orders of magnitude. For a low feedback resistance, the phase margin becomes small and peaking can be observed near $1 \mathrm{GHz}$. As the measured small-signal behavior is entirely due to the TIA, it can also be concluded that the photodiode responsivity is constant over the measured frequency band.

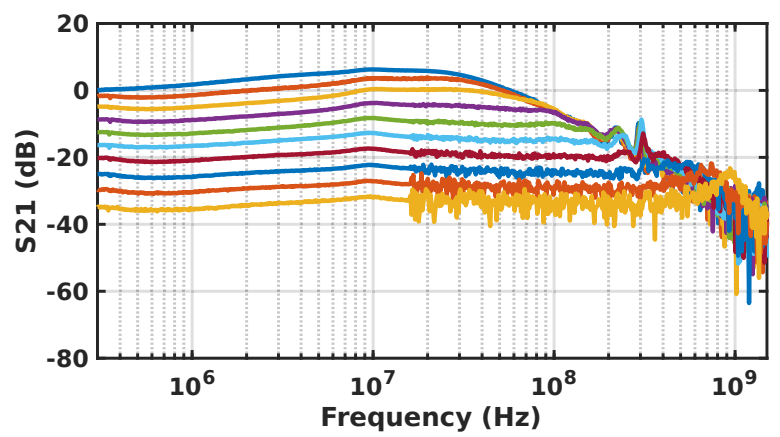

Fig. 6: Optical small-signal measurement results for different values of feedback resistance for $1550 \mathrm{~nm}$ light

Using a BER-tester, the receiver's $1310 \mathrm{~nm}$ light performance was analyzed, as can be seen in Fig. 7. For these measurements, a PRBS-7 data pattern was modulated onto $1310 \mathrm{~nm}$ light. As the Schottky photodiode responsivity for $1550 \mathrm{~nm}$ light is more than 3 times smaller than for $1310 \mathrm{~nm}$ light, the bit rate must be lower for this wavelength to obtain an open eye diagram. In Fig. 8, eye diagrams and their corresponding Q-factor can be seen for both 1310 and $1550 \mathrm{~nm}$ light. The Q-factor is defined as in (4) [8], where $\mu_{0 / 1}$ and $\sigma_{0 / 1}$ are the mean values and variances of the signal at the sample time for a digital 0 and 1 respectively and was calculated by the oscilloscope.

$$
Q=\frac{\mu_{1}-\mu_{0}}{\sigma_{1}+\sigma_{0}}
$$

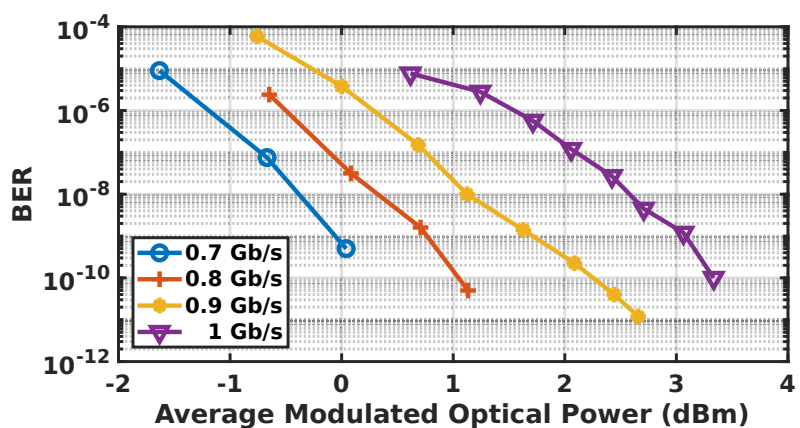

Fig. 7: Measured BER for $1310 \mathrm{~nm}$ light in function of average modulated optical power

As can be seen, for $1310 \mathrm{~nm}$ light, bit rates up to $1 \mathrm{~Gb} / \mathrm{s}$ are received at a BER of $10^{-10}$ for average optical signal power of $3.4 \mathrm{dBm}$, while eye diagrams with Q-factor higher than 20 have been achieved for $1550 \mathrm{~nm}$ light for bit rates up to $400 \mathrm{Mb} / \mathrm{s}$ for an average optical signal power of $1 \mathrm{dBm}$.

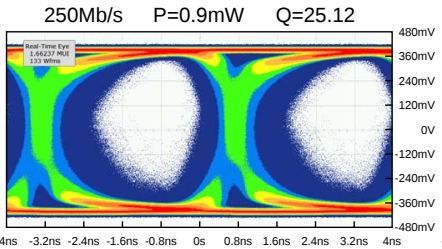

(a)

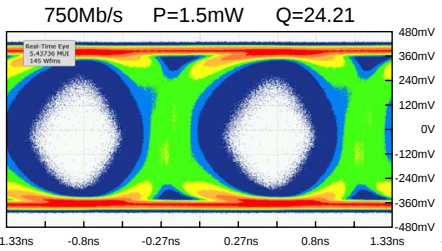

(c)

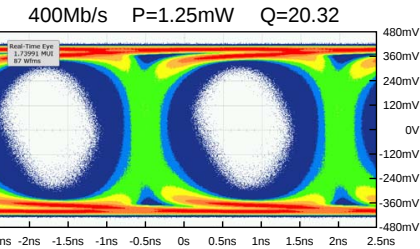

(b)

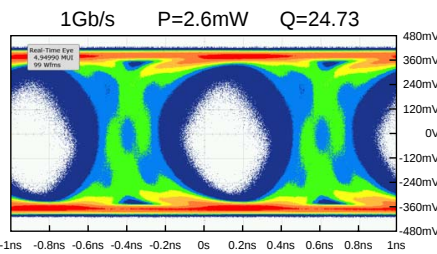

(d)
Fig. 8: Measured eye diagrams with Q-factor for PRBS-7 patterns. (a) $250 \mathrm{Mb} / \mathrm{s}, 1550 \mathrm{~nm}$ (b) $400 \mathrm{Mb} / \mathrm{s}, 1550 \mathrm{~nm}$ (c) $750 \mathrm{Mb} / \mathrm{s}, 1310 \mathrm{~nm}$ (d) $1 \mathrm{~Gb} / \mathrm{s}, 1310 \mathrm{~nm}$

\section{CONCLUSION}

The first 1310/1550 nm fully-integrated optical receiver in bulk CMOS has been presented. The low responsivity of the Schottky photodiodes can be overcome by the lownoise performance of the TIA, which was achieved by means of positive capacitive feedback. Measurement results have demonstrated that bit rates up to $1 \mathrm{~Gb} / \mathrm{s}$ are possible for $1310 \mathrm{~nm}$, while clean eye diagrams with high Q-factor have been acquired for $1550 \mathrm{~nm}$ light modulated up to $400 \mathrm{Mb} / \mathrm{s}$. This demonstrator paves the way for low-cost opto-electronics for these wavelengths.

\section{REFERENCES}

[1] D. Lee, J. Han, G. Han, and S. M. Park, "An 8.5-gb/s fully integrated cmos optoelectronic receiver using slope-detection adaptive equalizer," IEEE JSSC, vol. 45, no. 12, pp. 2861-2873, Dec 2010.

[2] J. S. Youn, M. J. Lee, K. Y. Park, and W. Y. Choi, "10-gb/s 850-nm cmos oeic receiver with a silicon avalanche photodetector," IEEE Journal of Quantum Electronics, vol. 48, no. 2, pp. 229-236, Feb 2012.

[3] J. Genoe, D. Coppee, J. Stiens, R. A. Vonekx, and M. Kuijk, "Calculation of the current response of the spatially modulated light cmos detector," IEEE ED, vol. 48, no. 9, pp. 1892-1902, Sep 2001.

[4] S. Radovanovic, A. J. Annema, and B. Nauta, "A 3-gb/s optical detector in standard cmos for 850-nm optical communication," IEEE Journal of Solid-State Circuits, vol. 40, no. 8, pp. 1706-1717, Aug 2005.

[5] W. Diels, M. Steyaert, and F. Tavernier, "Schottky diodes in 40nm bulk cmos for 1310nm high-speed optical receivers," in 2017 Optical Fiber Communications Conference and Exhibition, March 2017, pp. 1-3.

[6] W. Diels, M. Steyaert and F. Tavernier, "Modelling, design and characterization of schottky diodes in $28 \mathrm{~nm}$ bulk cmos for $850 / 1310 / 1550 \mathrm{~nm}$ fully integrated optical receivers," in 2017 47th European Solid-State Device Research Conference (ESSDERC), Sept 2017, pp. 224-227.

[7] W. Diels, M. Steyaert, and F. Tavernier, "Monolithic 1310nm 1gb/s optical receiver with schottky photodiode in 40nm bulk cmos," in 2018 Conference on Lasers and Electro-Optics (CLEO), May 2018, pp. 1-2.

[8] W. Freude et al., "Quality metrics for optical signals: Eye diagram, qfactor, osnr, evm and ber," in 2012 14th International Conference on Transparent Optical Networks (ICTON), July 2012, pp. 1-4. 